University of Nebraska - Lincoln

DigitalCommons@University of Nebraska - Lincoln

Faculty Publications, Department of Psychology

Psychology, Department of

2006

\title{
Mild Traumatic Brain Injury and Anxiety Sequelae: A Review of the Literature
}

Elizabeth L. Moore

Mayo, moore.elizabeth2@mayo.edu

Lori Terryberry-Spohr

Madonna Rehabilitation Hospital

Debra A. Hope

University of Nebraska-Lincoln, dhope1@unl.edu

Follow this and additional works at: https://digitalcommons.unl.edu/psychfacpub

Part of the Psychiatry and Psychology Commons

Moore, Elizabeth L.; Terryberry-Spohr, Lori; and Hope, Debra A., "Mild Traumatic Brain Injury and Anxiety Sequelae: A Review of the Literature" (2006). Faculty Publications, Department of Psychology. 592. https://digitalcommons.unl.edu/psychfacpub/592

This Article is brought to you for free and open access by the Psychology, Department of at DigitalCommons@University of Nebraska - Lincoln. It has been accepted for inclusion in Faculty Publications, Department of Psychology by an authorized administrator of DigitalCommons@University of Nebraska - Lincoln. 
REVIEW

\title{
Mild Traumatic Brain Injury and Anxiety Sequelae: A Review of the Literature
}

\author{
Elizabeth L. Moore ${ }^{1}$, Lori Terryberry-Spohr², \& Debra A. Hope ${ }^{1}$ \\ ${ }^{1}$ University of Nebraska-Lincoln, Lincoln, Nebraska, USA \\ ${ }^{2}$ Madonna Rehabilitation Hospital, Lincoln, Nebraska, USA
}

Article received January 10, 2005; accepted July 16, 2005.

\begin{abstract}
There is scattered but significant psychological and neuropsychological evidence to suggest that mild traumatic brain injury (mild TBI) plays a notable role in the emergence and expression of anxiety. Conversely, there is also empirical evidence to indicate that anxiety may exert a pronounced impact on the prognosis and course of recovery of an individual who has sustained a mild TBI. Although the relationship between mild TBI and anxiety remains unclear, the present body of research attempts to elucidate a number of aspects regarding this topic. Overall, the mild TBI research is rife with inconsistencies concerning prevalence rates, the magnitude and implications of this issue and, in the case of PTSD, even whether certain diagnoses can exist at all. This review obviates the need for greater consistencies across studies, especially between varying disciplines, and calls for a shift from studies overly focused on categorical classification to those concerned with dimensional conceptualization.
\end{abstract}

Keywords: Mild traumatic brain injury, Head injury, Anxiety

\section{Introduction}

With both mild TBI and anxiety disorders boasting dramatic prevalent rates in the US, a greater understanding of their interaction and its implications is paramount in the treatment of the potentially hundreds of thousands each year affected by this phenomenon. The primary goal of the current review is to compile and critically examine the limited research available on mild TBI and the functional and etiological issues associated with anxiety sequelae. The review begins with a 'crash course' on the definition and clinical picture of mild TBI, followed by a more in-depth examination of general anxiety symptoms precipitated by a mild TBI, as well as theories of the neuropsychology and etiology of acquired anxiety. In addition, the present literatureon post-traumatic stress disorder (PTSD), obsessive-compulsive disorder (OCD), generalized anxiety disorder (GAD), panic disorder (PD), specific phobia and social phobia is reviewed and followed by data regarding the impact of acquired anxiety on neuropsychological symptoms and functional outcome of those with mild TBI.

Correspondence: Elizabeth L. Moore, PhD, Mayo Clinic, OCD/Anxiety Disorders Program, Rochester, Minnesota, USA; moore.elizabeth2@mayo.edu.
Whenever possible, data specifically pertaining to mild TBI are made the central focus of this paper. However, due to a dearth of such research, data based on mixed TBI samples (those including mild, moderate and severe TBIs) are included when deemed appropriate and particularly relevant. When available, specific percentages of those with mild TBIs in such studies are provided. Although such circumstances are not ideal, data from mixed TBI samples arguably maintain a notable degreeof utility within mild TBI research. Evidence for a 'biogradient' (i.e. the more severe the injury, the more severe the symptoms) is inconsistent across studies, with many investigators actually reporting an inverse biogradient (those with mild TBI endorsing more psychiatric symptoms than those with moderate or severe TBIs) [1, 2]. It should also be noted that the present review is concerned with anxiety with an onset precipitated by a mild TBI. A diagnosis labelled 'acquired' can be assumed to have surfaced following a mild TBI, without recent injury pre-morbidity. Those with current morbidity of an anxiety disorder at the time of injury, although acknowledged in this review, are not a main focus.

\section{Mild traumatic brain injury}

Definition

Providing a clear definition of what constitutes a mild TBI is a task which proves more elusive that one would 
expect. In general, TBI refers to 'a sudden and very serious physical damage to the face, skull, scalp, dura or brain caused by a mechanical force that can produce devastating multiple psychosocial, cognitive and physical disabilities' ([3], p. 82). However, the fields of neuropsychology, neurology and related disciplines have struggled to reach a consensus on what specific clinical criteria constitute mild TBI [4]. This problem is an obvious detriment to the generalizability of present mild TBI research and, more importantly, to the patients who are in need of treatment for such injuries. A number of attempts at a uniformly recognized definition have been proffered by sources including, most notably, the Centers for Disease Control (CDC) and Prevention's Mild Traumatic Brain Injury Working Group [5] and the Mild Traumatic Brain Injury Committee of the American Congress of Rehabilitation Medicine (ACRM) [6].

According to the criteria delineated by the CDC [5], mild TBI is defined by 'the occurrence of injury to the head arising from blunt trauma or acceleration or deceleration forces with one or more of the following conditions attributable to the head injury:

- Any period of observed or self-reported:

o transient confusion, disorientation or impaired consciousness;

o dysfunction of memory around the time of injury; or

- loss of consciousness lasting less than $30 \mathrm{~min}-$ utes.

- Observed signs or other neurological or neuropsychological dysfunction, such as:

o seizures acutely following the injury to the head;

o irritability, lethargy or vomiting following head injury, especially among infants or very young children; or

o headache, dizziness, irritability, fatigue or poor concentration, especially among older children and adults' (p. 4).

The Mild Brain Injury Special Interest Group of the ACRM ([6], p. 86) has also proposed criteria for the diagnosis of mild TBI. Their definition and criteria are as follows: 'A patient with [a mild TBI] is a person who has had a traumatically induced physiological disruption of brain function, as manifested by at least one of the following:

(1) any period of loss of consciousness,

(2) any loss of memory for events immediately before or after the accident,

(3) any alteration in mental state at the time of the accident (e.g. feeling dazed, disoriented or confused), or
(4) focal neurological deficits(s) that may or may not be transient.'

Further, the authors indicate that a diagnosis of greater severity be given if loss of consciousness (LOC) persists longer than 30 minutes, if posttraumatic amnesia lasts (PTA) longer than 24 hours or if 30 minutes postinjury, a Glasgow Coma Scale (GCS) is assessed at less than 13. The GCS is a widespread prognostic indicator used in the early triaging of a TBI patient to determine whether neuroimaging or neurosurgery is warranted [4]. It consists of a scale ranging from 3-15, with higher scores indicating higher levels of functioning [7].

The CDC and ACRM definitions are generally in concert. Both require that LOC must last less than 30 minutes and specify that a state of disorientation or confusion, as well as memory dysfunction, can satisfy criteria without formal LOC. Although none of the criteria listed in each definition are at conflict with each other, there are differences in the focus of additional criteria provided. While the ACRM definition includes GCS scores as an extra determiner of severity, the CDC definition provides more detail regarding signs and symptoms following the injury. One of the CDC criteria also includes symptoms including irritability, dizziness, headache and poor concentration, which have also been included in the criteria of post-concussional syndrome (PCS), a provisional and yet to be standardized constellation of symptoms. Such symptoms represent a problematic overlap in the study of anxiety symptoms in those with mild TBIs. Many of the same symptoms caused by the physical damage of a mild TBI can also be caused by the increased heart and respiration rates, muscle tension and other physiological changes triggered with anxiety. This overlap can obviously lead to either the under-diagnosis of anxiety symptoms following mild TBI and an inflated sense of the physical damage caused by the injury. It is crucial, therefore, for mild TBI criteria to account for this issue to ensure the appropriate diagnosis and most effective intervention for the injury and/or resulting anxiety.

\section{Prevalence}

Mild TBI has been termed the 'silent epidemic' of our times [8]. An estimated 1.5 million people in the US alone suffer a mild TBI each year, representing 395,000 hospitalizations annually $[9,10]$. Mild TBI encompasses the vast majority of all TBIs, representing $75-80 \%$ of all head-injured patients $[9,10]$. Approximately $25 \%$ of those with a mild TBI are hospitalized, 35\% were treated in an emergency department and released, $14 \%$ received outpatient treatment and $25 \%$ received no medical care [9]. Motor vehicle accidents are thought to account for upwards of $45 \%$ of mild TBIs, with falls $(30 \%)$, occupational accidents $(10 \%)$, recreational acci- 
dents $(10 \%)$ and assaults $(5 \%)$ comprising the remaining injuries [11].

\section{Risk factors}

The most significant risk factor is for young men between the ages of 15-24 [10]. Males sustain mild TBIs two-to-three times more frequently than females, with motor vehicle accidents, assaults and gunshot wounds accounting for the huge gender discrepancy [11, 12]. Other recognized risk factors include alcohol consumption and other substance abuse, lower socioeconomic levels, living in congested urban areas and a history of marital discord, learning disability or previous TBI [11]. Pre-injury psychiatric difficulties have also been cited as having an association with mild TBI. Significantly higher percentages of psychiatric inpatients have reported a history of TBIs at rates exceeding the $5-24 \%$ reported in the general public [11]. A study conducted by McGuire et al. [13], for example, cites a rate of $36 \%$ of evaluated inpatients reporting a history of TBI with LOC $(n=231)$. Another study found the $68 \%$ of the 100 psychiatric inpatients had incurred a TBI [14]. Although some list psychiatric difficulties as a formal risk factor, only an association and no clear causal connection can be assumed.

\section{Prognosis and recovery}

For the majority of mild TBI cases, a good recovery can be expected [15]. Those with a good outcome typically recover over a relatively short period of time, with PCS symptoms dissipating over several weeks to 3 months for most cases [16]. For a number of patients, however, symptoms linger for several more months or even years. An estimated $51 \%$ of patients have at least one symptom after 6 weeks and 15\% still report symptoms at 1 year post-injury [17]. Complaints have even been documented 5 years post-injury and as long as 23 years post-injury, with many investigators suggesting that impairment in some may be permanent [10]. In cases of such long-term impairment, the typically reported mitigation of symptoms over time may actually represent a behavioral adaptation as opposed to a legitimate return to pre-injury levels of functioning [10]. It should be noted that, again, because of the overlap between PCS and anxiety symptoms, these lasting symptoms could be representative of the untreated mental health symptoms themselves rather than simply remnants of the physical injury.

An individual's recovery may be impacted by a wide range of factors such as concurrent dementia, chronic alcohol and/or drug effects, advanced age, borderline intellectual functioning, medication side-effects, preexisting psychiatric conditions or personality factors and social or demographic factors which interact with the injury circumstances $[10,11]$. Post-injury culprits include new psychiatric conditions occurring since the injury and, for some, the influences of compensation and litigation factors [10].

\section{Anxiety and mild TBI}

\section{Prevalence and description}

Among the general population, anxiety disorders collectively have the highest prevalence of any other group of disorders and have been called 'the single largest mental health problem in the country' ([18], p. 22). Lifetime occurrence is reported at $29 \%$ [19]. A growing body of research indicates that anxiety disorders may be even more prevalent in the aftermath of a mild TBI. Anxiety in general has been reported at rates as high as $70 \%$ in participants with TBIs [12]. A meta-analysis of 12 studies, comprising 1199 total participants, revealed an overall prevalence of anxiety disorders as $29 \%$ across all severity of TBI [20]. When moderate, severe and undifferentiated TBI studies are excluded, that rate drops slightly to $23 \%$ for mild TBI (over three studies). This estimate is supported by a more recent study by Mooney and Speed [15] in which $24 \%$ of their participants with mild TBIs were classified as having developed an acquired anxiety disorder. Although some are much more prevalent than others, virtually all types of anxiety disorder have been documented following mild TBI. Research indicates that the rates of anxiety disorders among patients with TBI are $3-28 \%$ for GAD, $4-17 \%$ for PD, $1-10 \%$ for phobic disorders, 2-15\% for OCD and 3-27\% for PTSD [21, 22]. Currently, such acquired anxiety disorders are presently coded in the DSM-IV-TR as 'anxiety disorder due to a medical condition, with no indication of severity' [23]. In general, the most common post-TBI anxiety symptoms include free-floating anxiety, fearfulness, intense worry, generalized uneasiness, social withdrawal, inter-personal sensitivity and anxiety dreams [12].

\section{Neuropsychology of anxiety}

Gray and McNaughton [24] present a complex model of anxiety by integrating neuropsychological mechanisms, the function of specific brain structures and their combined contribution to the varied manifestations of anxiety. They postulate that activity in a 'behavioral inhibition system' in the brain produces anxious symptoms. This sophisticated system is comprised mainly of the septo-hippocampal system, but also includes, among other areas, the anterior thalamus, 'Papez circuit', cingulate cortex, pre-frontal cortex and ascending noradrenergic fibres of the locus coeruleus. Anxiety becomes a chronic problem when its correlating brain areas within the behavioral inhibition system malfunction and become overly sensitive to stimuli. For example, OCD is said to arise when the septo-hippocampal 
system, whose job it is to check one's environment for aversive or novel stimuli, becomes overly sensitive to certain stimuli, reacting too frequently. This results in the persistent checking and searching that are so often symptoms of OCD [25]. In sum, individuals who are particularly vulnerable to anxiety have excessively reactive behavioral inhibition systems. The range of anxiety disorders simply represents different types of reactivity within the nuances of the system and the specific stimuli or sets of stimuli involved.

Although Gray's and McNaughton's model pinpoints focal areas of the brain, mild TBIs tend to be the result of more diffuse damage. Even though the theorists do not specify how the septo-hippocampal system and its related brain structures might be impacted by mild TBI, it is possible to speculate about this, using what is known about the nature of the damage typically inflicted by a mild TBI. For example, motor vehicle accidents account for almost half of all mild TBIs [11]. During such accidents, acceleration-deceleration forces and the acute movement of the head can result in focal cortical contusions, abrasions on the surface of the brain from direct contact with the skull (a coup injury) [26]. Abrasions may also result from contact to the skull across from the initial point of impact as the brain 'rebounds' (a contrecoup injury) [26]. The pre-frontal cortex, which relays environmental, verbal and predictive information to the septo-hippocampal region [24], is in a prime position to sustain focal abrasions from either coup or contrecoup injuries. With the septo-hippocampal system safely located deep within the limbic system of the brain, such superficial abrasions are less likely to cause it damage. The limbic system, however, has proven particularly vulnerable to deeper lesions, which are often the result of rotational forces which produce the shearing of axons [27]. Such injuries are in no way limited to motor vehicle accidents and can be incurred in an array of other situations including falls, occupational and sports-related accidents and assaults. Although no direct investigation exists, it seems reasonable to conclude that Gray's and McNaughton's 'behavioral inhibition system' could be a frequent recipient of mild TBI damage, in part because of its complex and widespread pathways throughout the brain.

\section{Etiology}

It is generally accepted that acquired psychiatric conditions can trace their etiology to organic causes, psychogenic origins or an interaction of the two [28]. Many individuals who have sustained a mild TBI have experienced life-threatening events and overwhelming stressors. Both immediate stressors, such as hospitalization or PTA, and more long-term stressors, such as the gradual realization of possible permanent impairment, have the potential to impact an individu- al's mental health. Additionally, those with a TBI must also face an array of hurdles such as loss of job, chronic pain, social isolation, memory loss, financial difficulties and litigation [20]. For example, Lezak [29] reported that $76 \%$ of her TBI sample $(n=42)$ demonstrated distractibility, fatigue and perplexity. Such symptoms were strongly linked to pathological worry and anxiety among her participants.

Although this review is specifically concerned with those who have acquired an anxiety disorder strictly following a mild TBI, it should be acknowledged that a large number of those who sustain a TBI of any severity have pre-existing psychiatric conditions [16]. In fact, as previously mentioned, psychiatric history has a significant association with mild TBI [13]. It is speculated that higher rates of substance abuse account for some of this increased vulnerability, but little else has been hypothesized. Further research is needed to elucidate other underlying risk factors of this trend. Another peripheral set of circumstances which should be mentioned is the reactivation of previous psychiatric conditions that have been 'dormant' for an extended period of time [16]. Mild TBI is thought to break down psychological defenses and formerly effective coping strategies, leaving one vulnerable to previously experienced anxiety conditions [30].

Aside from those related to Gray's and McNaughton's anxiety model [24], other organic factors in anxiety etiology have received attention from investigators. Although conclusive evidence has yet to be documented, several studies indicate that localization of brain injury may play a role in the psychiatric sequelae, including anxiety, of a TBI. Traditionally, investigations have preferred participants with penetrating head injuries which allow for cleaner localization of the lesion [31]. A variety of such studies have found associations between right orbital cortex, left occipital lobe and temporal lobe injuries and the regulation of anxiety [20]. Evidence also indicates that even much more general lateralization of a TBI may influence the degree and nature of anxiety sequelae. Anxiety has been reported to be a common feature of left-hemisphere damage and is manifested as over-sensitivity, excessive cautiousness and exaggerated appraisal of one's own impairment [20]. Conversely, participants with brain injuries localized in the right hemisphere, including those with mild TBIs, have been reported to demonstrate fewer anxiety symptoms that would be expected, often exhibiting indifference and lack of insight in their place $[20,31]$.

\section{Anxiety symptoms and mild TBI}

The vast majority of studies on this topic have focused on categorical diagnoses rather than levels of general anxiety symptoms. As discussed later in this 
review, categorical diagnoses are limited to the extent that the diagnoses themselves are valid constructs. In the case of anxiety sequelae precipitated by a mild TBI, traditional diagnoses may be less valid than for those in the general population, given the complexity of factors involved in their presentation, many of which are poorly understood. Quantitative measurement of symptoms would likely increase the amount of clinical information available, boost reliability and lead to more clinically relevant data on outcome and treatment [32]. A handful of studies have ventured into this realm. Schoenhuber and Gentilini [33], for example, used the State-Trait Anxiety Inventory (STAI) [34] to measure both state anxiety (anxiety present at the time of testing) and trait anxiety (anxiety in the form of a personality trait) in a sample of participants with mild TBIs and matched controls [33]. Surprisingly, those with mild TBIs exhibited scores on both state and trait sub-scales that were not significantly different than their matched controls. The authors suggest that the STAI may not be sensitive enough to be of much clinical utility in mild TBI populations. Other studies [35, 36] have made use of the MMPI [37], an inventory that provides a profile of an individual's level of psychopathology based on 10 clinical scales [38]. Unfortunately, although the MMPI features an anxiety scale, it is a supplementary scale [38] and has not yet been included in authors' interpretations of clinical scales or present in data analyses. The MMPI has repeatedly demonstrated its utility in mild TBI populations [35] and it is hoped that future research will expand its scope to include the anxiety sub-scale of the MMPI. Additional measures such as the Beck Anxiety Inventory [39] and the anxiety, avoidance and PTSD scales of the Millon Clinical Multiaxial Inventory-III [40] are additional quantitative measures of anxiety and its correlates which may help to bolster the data available on the levels of anxiety sequelae following mild TBI.

\section{Depression and anxiety}

No comprehensive review of anxiety and mild TBI would be complete without an acknowledgment of comorbid depression. While primacy may vary, depression and anxiety are notorious for their high degree of co-morbidity, with reported rates ranging from 33$65 \%$ [41]. Adding mild TBI to the mix does nothing to ameliorate the situation and may, in fact, increase this phenomenon. For example, Jorge et al. [42] evaluated a mixed TBI sample, $15 \%$ of whom had sustained a mild TBI. One hundred per cent of those diagnosed with GAD also met criteria for major depression $(n=7)$.

The vast majority of mild TBI research focuses primarily on anxiety or depression alone or, when evaluating prevalence rates for both, does not provide comorbidity data. When available, specific data on co- morbidity rates of depression will be provided in the following sections. Given the small scale of many of the studies, the number of subjects in each is noted. Additionally, the number of studies specifically focused on each disorder is included. The number indicated does not include the six prospective studies concerned with prevalence across Axis I disorders nor the four literature reviews or chapters which have compiled and summarized the extent research on anxiety disorders and TBI.

\section{Specific anxiety disorders and mild TBI}

\section{Post-traumatic stress disorder}

Post-traumatic stress disorder (PTSD) is characterized by the re-experiencing of an extremely traumatic event, usually by way of nightmares and intrusive thoughts of the incident. In addition, symptoms of heightened arousal and avoidance of stimuli associated with the trauma must be present [23]. Such symptoms must be present for more than 1 month and cause significant distress or impair the individual's functioning. When an individual manifests such symptoms for a period of time for less than 1 month, he or she would be assigned a diagnosis of Acute Stress Disorder. Lifetime prevalence of PTSD in community-based studies is $6.8 \%$ [19]. The nature of trauma varies widely, from natural disasters to rape. Thirty-nine per cent of traffic accident victims, $15 \%$ of Vietnam veterans and $24 \%$ of young urban adults meet criteria for PTSD [43].

\section{PTSD and mild TBI}

Given the often violent and life-threatening circumstances of mild TBI, PTSD is arguably the most expected of all anxiety sequelae. It is, without a doubt, by far the most studied; no fewer than 58 studies are currently available in the literature. Prevalence rates, however, vary notably by study. Bryant and Harvey [44] reported a frequency of $20 \%$ in a sample of motor vehicle accident victims who had sustained mild TBI $(n=46)$. Feinstein et al. [45] reported that $84 \%$ of participants with mild TBI were assessed as having PTSD symptoms $(n=57)$. No formal diagnoses were attempted, but it was noted that the evaluation involved a scale highly correlated with formal diagnosis of PTSD. As compared to participants with brain injuries who did not have PTSD, those with PTSD have been found to be significantly more depressed and generally anxious. Many who suffer PTSD post-injury continue to experience symptoms for several months, even years. A PTSD rate of $13 \%$ in a mild TBI sample at 3 months post-injury was reported by Levin et al. [46] $(n=60)$. Additionally, $18 \%$ of those with PTSD met criteria for co-morbid major depression. Harvey and Bryant [47] found that $24 \%$ of their participants with mild TBIs 
met criteria for PTSD at 6 months post-injury $(n=48)$. This rate held relatively steady at $22 \%$ when the sample was re-assessed at 2 years post-injury.

Prominent PTSD symptoms of those with TBI may differ from those in community samples in which intrusive thoughts are generally reported to be the dominant symptom. Ohry et al. [48] found dreams and nightmares, in addition to hyper-arousal, to be the most commonly endorsed symptoms among their sample of undifferentiated TBI severity, $33 \%$ of whom were diagnosed with PTSD $(n=24)$. This unique symptom presentation was attributed to the often spotty recollection of the traumatic event by participants. Even when memories are not encoded due to amnesia or LOC, some researchers assert that an increased arousal in situations that are similar to the trauma should satisfy the re-experiencing criterion. Turnbull et al. [49] obtained data regarding PTSD symptoms from a sub-sample of 13 participants with mixed TBI severities who had no recollection of their respective traumatic events. The authors reported that the most frequently experienced intrusive symptoms were psychological and physiological distress in response to cues related to the traumatic event. Warden et al. [50] suggest modified criteria for individuals with TBI by excluded re-experiencing symptoms. Implementing the modified criteria identified six individuals in their sample of 47 participants with moderate TBI who had been overlooked by the Diagnostic and Statistical Manual, 3rd Edition Revised (DSM-III-R) [51] criteria. None of the sample met the standard criteria.

A number of factors has been implicated in increasing one's vulnerability to PTSD following TBI. Feinstein et al. [45] concluded that substance abuse, the presence of co-morbid depression or anxiety disorders and the added stress of litigation following the injury increase one's vulnerability. In addition, those with fatigue, dizziness, headache and pain as opposed to those without reported a significantly greater number of PTSD symptoms, despite having TBIs of similar severity [45]. After assessing a sample of participants with mild TBI, Harvey and Bryant [47] found that increasing age, a history of PTSD, BDI score and an avoidant coping style increased one's risk for developing acute symptoms of a stress response, a pre-cursor to PTSD. In a study involving those with severe TBI, Bryant et al. [52] found that an avoidant coping style, behavioral coping style (vs. cognitive coping style) and prior unemployment were significant predictors of the development of PTSD and its severity $(n=96)$. It was noted that the prior unemployment predictor was representative of pre-injury functioning, keeping in mind that pre-morbid level of functioning, including physical illness and poverty, have been shown to be correlated with the development of PTSD in the general population [52]. Stud- ies of mixed TBI samples have also implicated a history of depression in first-degree relatives and history of PTSD from prior trauma in increased vulnerability to developing PTSD post-TBI ( $n=47$ and $n=158$, respectively) $[50,53]$.

Presence of PTSD with loss of consciousness and post-traumatic amnesia

PTSD following TBI presents a notable controversy within the literature. Many argue that LOC and PTA are protective mechanisms which preclude the development of post-traumatic symptoms triggered by the traumatic event $[54,55]$. Others, however, argue that PTSD is a frequent follower of TBI, with studies documenting rates as high as $50 \%$ [56].

PTA and LOC: Definitions. PTA and LOC have been called 'the hallmark feature for diagnosing a mild TBI' ([4], p. 944). PTA is defined by the ACRM as 'any loss of memory for events before and after the event' ([57], p. 106). Patients with PTA typically experience a period of retrograde amnesia which may render the individual amnestic not only for the injury itself, but also for the minutes or hours prior to the event. Although a formal LOC of varying lengths of time is required to meet inclusion criteria in many studies, the CDC and ACRM consider any period of altered mental state (e.g. confusion, disorientation, or impaired consciousness), sufficient for diagnosis of a mild TBI ([5], [6]), [57], p. 106). Therefore, the debate concerning LOC and PTA bear a large impact on what is considered to qualify as a mild TBI in the present literature.

Evidence against the presence of PTSD. In 1942, Adler [58] published a study examining post-traumatic symptoms in victims who had lost consciousness in the Coconut Grove night club fire and the ensuing stampede. Adler evaluated 54 participants and determined that, of the 20 who reported no psychiatric consequences, 15 had also experienced LOC. He concluded that LOC had a direct and preventative impact on the development of post-traumatic sequelae. The nature of this study, although not directly involving TBI, easily lends itself to such a context and has set the stage for investigation specifically addressing the development of PTSD in individuals who had experienced PTA. Those who argue against the presence of PTSD assert that individuals with no memory for the traumatic event are exempt from the hallmark and one of the necessary criteria of PTSD: re-experiencing the traumatic event, such as through intrusive thoughts or nightmares [50, 54, 55]. Sbordone and Liter [54] compared participants with no brain injuries who had been diagnosed with PTSD with a group of 70 participants with mild TBI. While the PTSD group was able to vividly describe their 
traumas, including emotionally-charged details, none of the mild TBI participants were able to do so. In addition, none with mild TBI were reported to have exhibited distress when discussing their trauma, nor did they report typical PTSD symptoms such as intrusive memories, heightened arousal or nightmares. The authors concluded that 'PTSD and mild TBI are mutually incompatible since patients who sustain PTSD simply cannot "forget" the traumatic event, whereas patients who sustain mild TBI have no recollection of the traumatic event' ([54], p. 411).

Warden et al. [50] evaluated 47 active-duty soldiers who had received moderate TBIs with resulting LOC with or without PTA. None of their participants could recall the traumatic event. Although $13 \%$ of the participants reported symptoms such as hyper-sensitivity, a heightened startle reflex, irritability, difficulty concentrating and avoidance of stimuli related to the trauma, none reported re-experiencing the trauma and, therefore, none met full DSM-III-R [51] criteria for PTSD. The authors speculate the lack of intrusive memories and re-experiencing symptoms in their sample was a direct result of PTA. They conclude that TBI accompanied by PTA and co-morbid PTSD is very uncommon.

Evidence for the presence of PTSD. There exist a number of studies, however, that contend that PTSD can exist in the absence of an overt memory for a traumatic event and attempt to supply evidence that emotional reactions to trauma can be retained without conscious recall. Indeed, Bryant [59] has suggested that those with mild TBI and LOC or PTA may develop 'pseudomemories' which are analogous to the flashbacks which would otherwise be experienced in PTSD. Bryant provides a description of two participants who had sustained closed head-injuries in motor-vehicle accidents and developed delayed-onset PTSD. Although both participants were amnestic for their traumas (one having PTA lasting a full 5 weeks), both experienced pseudomemories of the events based on secondhand information, such as police reports and newspaper coverage and subjectively generated images. These pseudomemories were intensely vivid and intrusive and were accompanied by other symptoms of PTSD including heightened startle response, hypervigilence, sleep disturbance and avoidance of stimuli related to the trauma (driving). Bryant states that the present conceptualization of PTSD does not appreciate that 'traumatized individuals can develop representations of a traumatic event ...and that these representations can be experienced as involuntary and subjectively compelling' ([59], p. 626).

An additional hypothesis has been presented by King [60], by way of a case study involving a man who had been hit by a car while hitch-hiking and who had sus- tained a mild TBI. Despite a 2.5 day period of PTA, he met full criteria for PTSD by virtue of his reports of intrusive 'islands' of memory for the incident. Such islands of memory are defined as 'a recollection of events which occur outside continuous memory for events' (p. 82) and are reported to be present in at least one third of all mild TBI injuries [60]. They often involve memory fragments of events such as being carried into the ambulance or transported to the hospital [61]. King postulates that 'islands' of memory occur when 'the notable heightened state of arousal present immediately after [an] injury [is] sufficient to generate declarative memory of the event which would not have been possible due to post-traumatic amnesia under less traumatic circumstances' ([61], p. 83).

Turnbull et al. [49] evaluated a sample of mixed TBI participants, $56 \%$ of whom had sustained a mild TBI. Prevalence rates for PTSD were reported at a range of $17-27 \%$ taking into account differing scoring criteria (lenient vs. stringent) for each participant $(n=53)$. Twenty-six per cent had no memory of the incident (6\% of whom had mild TBIs), $25 \%$ had an untraumatic memory ( $23 \%$ mild) and $49 \%$ had a traumatic memory ( $30 \%$ mild). Increased psychological distress was correlated with having traumatic or no memories of the event. The presence of untraumatic memories produced little in the way of psychological distress following a head injury. The authors conclude that having amnesia for a traumatic event, although not protective against PTSD, does seem to be related to a decreased severity of symptoms and is particularly protective against the intrusive symptoms of PTSD.

\section{Comment on the literature}

To date, there has been no conclusive answer as to whether conscious recollection of a trauma is essential in the development of PTSD [22]. This debate has clearly spawned a number of elucidating studies which may indirectly serve to engender a greater understanding of the mechanisms underlying post-traumatic reaction to trauma. They also, however, raise questions regarding the present conceptualization of PTSD. Research on this topic has held firm the criteria laid out in the DSM, important for generalizable data for those using the same criteria, but perhaps deleterious to the patients whose symptoms, sometimes quite severe, may be illegitimized by a technicality. Depending on the ultimate goal of ongoing research, it may be beneficial for future investigators to shift their focus from qualitative diagnosis to quantitative measurement of symptoms. As opposed to the categorical system that presently dominates the research, the present vein of investigation might benefit from the use of a dimensional system which 'classifies clinical presentations based on quantification of attributes rather than the assignment 
to categories' ([23], p. xxxii). Although it was not their stated intent, studies such as the one carried out by Feinstein et al. [45] measure symptoms rather than diagnosing individuals and dismissing those who do not fit full criteria. This quantification of symptoms tends to increase the amount of clinical information available and boost reliability [32].

Also, despite the flurry of research fixated on the importance of conscious recollection of a trauma, none have investigated whether differences in symptom severity, functional outcome, etc. exist between those who meet full criteria for PTSD and those who lack recall of the trauma but otherwise achieve full criteria. The answer to this question may render this conceptual debate moot and perhaps fuel a reconsideration of what constitutes a clinically significant reaction to a traumatic event.

\section{Obsessive-compulsive disorder}

Obsessive-compulsive disorder (OCD) is characterized by recurrent obsessions and compulsions which cause notable impairment of functioning and are significantly time consuming [23]. Obsessions are intrusive thoughts or images often concerned with contamination, doubts about having carried out a task (e.g. turning off the stove), order and organization and horrific or aggressive impulses [23]. Compulsions are repetitive behaviors or mental acts, such as praying or counting, that are designed to relieve the anxiety of a preceding obsession [23]. Typically having its onset in late adolescence and early adulthood, OCD is reported to have a lifetime prevalence rate [19].

\section{OCD and mild TBI}

Reported prevalence rates of OCD following TBI have varied widely, with some reporting rates consistent with those found in community samples. In a meta-analysis of three early studies, Epstein and Ursano [20] note that $3 \%$ of those evaluated were found to have OCD (total sample of 759 participants of unspecified TBI severity). Deb et al. [62] report a modest rate of $1.6 \%$ in their sample of mixed TBIs $(n=196$ participants; 58\% with mild TBI) 1 year post-injury. Others, however, cite OCD rates at much more elevated. Fourteen per cent of a sample with mixed TBIs evaluated by Hibbard et al. [7] met criteria for OCD $(n=100)$. van Reekum et al. [63] found a rate of $11 \%$ of a sample of those with mixed TBIs ( $28 \%$ mild) at least 2 years postinjury $(n=18)$.

In addition to the aforementioned studies, this vein of research features a number of smaller-scale studies, with a primary focus on mild TBI. Kant et al. [64] evaluated four participants who had sustained mild TBIs and had subsequently developed OCD. The authors point out that, as opposed to the typical focus on dis- crete brain lesions and OCD, it is quite possible to develop OCD following minor and diffuse damage to cerebral tissue. McKeon et al. [65] also examined four individuals who had sustained TBIs, three of whom had TBIs of mild severity. The authors note that, in each case, increased emotional arousal in the form of agitation and anxiety preceded the onset of OCD symptoms. They conclude that head injury is a probable contributor to the development of obsessive-compulsive neurosis; that although unusual, it is not a rare contributor' (p. 192).

OCD has also been reported to have surfaced after a notable delay following a TBI. Drummand and Gravestock [25] carried out a case study involving a man who had sustained a mild TBI during a violent work accident. The participant reported that, upon his return to work, roughly 6 months post-injury, he became highly pre-occupied with checking rituals to prevent selfharm as well as washing and hygiene compulsions at home. Consistent with McKeon et al.'s finding, the participant experienced increased emotional arousal, including insomnia, irritability and headache, preceding the onset of these OCD symptoms. The authors cite the previously detailed model presented by Gray and McNaughton [24] as a possible explanation for the man's clinical presentation. Drummond and Gravestock postulate that this increased arousal may have resulted in his septo-hippocampal region becoming overly sensitive to stimuli and labelling formerly neutral stimuli as aversive. It is unclear, however, if his specific injury played a role in this process or whether it was precipitated by environmental or biological vulnerability factors independent of or interacting with his mild TBI. The authors do not speculate.

It has been asserted that the development of OCD following TBI may be related to the ability of an individual to cope with the consequences of his or her injury [20]. For example, compulsions may aid an individual in gaining a greater sense of control over one's life, a life which may seem confusing and disorganized to one who is attempting to cope with newly acquired neuropsychological impairment [20]. Others have focused their attention on a more organic conceptualization of OCD precipitated by mild TBI, capitalizing on neuroimaging and focal brain lesions. Although such research has not provided strong conclusive evidence for discrete lesion location, a number of areas in the brain have been implicated in playing a role in onset and maintenance of OCD symptoms $[22,66]$. Indirect evidence of organic etiology includes findings that individuals who develop OCD following a TBI have a negative family history of the disorder and later age of onset as compared to those without TBI $(n=13)$ [66].

Although the number of OCD-focused studies render it the second most studied anxiety disorder in popula- 
tions with TBI, the existing research only scratches the surface. Of the 18 studies available, 13 qualitatively examine case-studies, three are review articles and two are small-scale prospective studies. Clearly, the current body of literature falls short of providing a wellrounded and generalizable picture of this phenomenon. Brain scans have provided a provocative and partially convergent view of this phenomenon on a neurophysiological level. There is a great need, however, for much larger-scale studies. With such piecemeal data, from one case-study to the next, each using different protocols and measures, it is difficult to truly examine the nature of acquired OCD. Although such studies provide rich descriptions of this phenomenon, their generalizability and reliability and consequently their practical utility are highly compromised. Also, as previously mentioned, studies featuring quantitative data regarding symptoms rather than qualitative descriptions would greatly improve one's understanding of this unique presentation of OCD. There may be a different presentation of symptoms than found in other populations, data that could help to inform the treatment of such individuals. Larger-scale studies concerning the effective treatment of OCD following TBI are in particular need. If individuals do experience a notable change in their emotional arousal, as found in two of the case-studies, it may be possible to intervene at early signs of OCD and prevent its development. With continued research, it may be possible to predict those particularly vulnerable to acquired OCD, making proactive intervention more feasible.

\section{Panic disorder}

Individuals with PD experience recurrent and unexpected panic attacks with accompanying concern and anxiety regarding their occurrence [23]. To be recognized as having had a panic attack as defined in the DSM-IV-TR, an individual must experience at least four out of a list of 12 symptoms, including shaking, dizziness, chest pain, smothering sensations and fear of dying [23]. PD is frequently accompanied by agoraphobia, a disorder characterized by 'anxiety about, or avoidance of, places or situations from which escape might be difficult (or embarrassing) or in which help may not be available in the event of having a panic attack or panic-like symptoms' [23, p. 433]. Lifetime prevalence of PD community samples is reported at $4.7 \%$ [19]. The onset for PD typically occurs between late-adolescence and mid-30s and can be triggered or exacerbated by stressful life events such as divorce or death of a loved one [23].

\section{PD and mild TBI}

Despite the high prevalence of PD found in studies of those with TBI, it has been paid little attention in the literature. Save one single case study [67], the only information on this phenomenon is derived from studies examining prevalence rates of Axis I disorders in general, with none specifically focused on PD. Deb et al. [62] found a 1-year prevalence of $9 \%$ in a sample of mixed TBI. Interestingly, the authors hypothesize that, in those with no memory for the traumatic event, PD may be an atypical expression of PTSD. Thirteen per cent of the mixed TBI sample studied by Hibbard et al. [7] met criteria for a diagnosis of PD (proportion of mild TBI not provided). Fann et al. [68] reported a post-injury onset rate of $4 \%$ among their mixed TBI sample $(n=50,58 \%$ of the sample had a mild TBI). Half of those diagnosed with PD had co-morbid agoraphobia. The notably lower prevalence rate in Fann et al.'s sample was likely due to the remarkably high preinjury rate of the sample. Ten per cent of their sample had pre-morbid PD and were excluded from the final tally of post-injury PD.

Although the present literature on mild TBI and PD provides some groundwork for the topic, many questions have yet to be answered. Considering the high co-morbidity rate of PD and agoraphobia, it is curious that the Fann et al. study was the sole study to mention or measure the occurrence of agoraphobia. It would seem possible for neuropsychological symptoms to influence the prevalence of agoraphobia accompanying PD. Depending on their expression, a range of symptoms such as attention and memory impairment, as well as judgement and insight issues, could have the potential to either elevate or decrease the prevalence of PD. Also, hypotheses of the etiology of acquired PD are virtually non-existent in the literature. If PD is indeed a manifestation of amnestic PTSD, a more detailed examination of the course of post-injury status is essential to identify potential differences in course and clinical picture. A greater understanding of this idea could inform both PTSD and PD research in the general population.

\section{Generalized anxiety disorder}

A diagnosis of GAD requires 6 months or more of excessive anxiety and worry about a number of activities or events in one's daily life [23]. In addition, symptoms such as muscle tension, restlessness, sleep disturbance or concentration difficulties must be present. In community samples, lifetime prevalence is 5.7\% [19]. Women are at much greater risk for developing GAD, outnumbering men at a rate of two-to-one [69]. GAD has been found to frequently co-exist with other psychiatric disorders, most commonly major depression [23].

\section{GAD and mild TBI}

GAD following TBI has been reported at rates, on average, that are double those found in the general popu- 
lation [22]. Rates of acquired GAD, however, have varied. Hibbard et al. [7] reported a prevalence rate of $8 \%$ for GAD developed following a TBI. Unfortunately, the descriptives provided do not allow for differentiating among injury severities. Deb et al. [62], however, reported a prevalence of $2.5 \%$ among their mixed TBI sample, a rate that is slightly less than that of general population samples. The sample was evaluated 1 year following the onset of the injury which may account for the disparity in reported GAD rates.

Surprisingly, no studies to date have investigated the prevalence of GAD using a strictly mild TBI sample. The few studies, two in all, that have investigated the occurrence of acquired GAD feature samples of mixed TBI severity. Two studies have focused on the frequency of GAD in those with a TBI as well as its relationship to major depression. In a study conducted by Fann et al. [68], 24\% of 50 consecutive outpatients with TBI were diagnosed as having GAD (58\% with a mild TBI). GAD rates, unfortunately, were not broken down by injury severity. Interestingly, $71 \%$ of the depressed/ anxious group in the study (those with GAD and comorbid major depression) possessed a mild TBI. Jorge et al. [42] evaluated 66 consecutive patients admitted to a shock trauma centre, $15 \%$ of whom had sustained a mild TBI. Seventeen per cent of those with a mild TBI were diagnosed with GAD. As previously mentioned, $100 \%$ of those diagnosed with GAD also met criteria for major depression.

No study to date has examined GAD with respect to specific organic etiology. Nor have any addressed the etiology of GAD in terms of psychogenic factors. With such a high co-morbidity with major depression, GAD may, in some way, be a product of major depression. Some researchers, in fact, have hypothesized that comorbid anxiety and depression may represent a syndrome phenomenologically different than depression or anxiety alone [41]. This said, investigation into the differences between those with major depression alone and those with co-morbid GAD following mild TBI may be a worthwhile avenue to pursue.

\section{Specific phobia}

Specific phobia is defined by clinically significant anxiety brought on by exposure to a specific feared object or situation, such as insects, heights, confined places or receiving injections [23]. The feared stimulus is often avoided and can lead to significant interference in one's life [23]. Most frequent ages of onset are during childhood and in an individual's mid-20s. Lifetime occurrence is cited as $12.5 \%$ [19].

\section{Specific phobia and mild TBI}

Limited research involving specific phobias precipitated by mild TBI or any TBI has been conducted. Ex- isting data based on the only three available studies, with only one specifically focused on the topic, reveal few incidences of specific phobias following TBI. Deb et al. [62] noted that only one participant of the 196 evaluated met criteria for a specific phobia, a rate of $0.8 \%$. One out of 18 participants of mixed TBI studied by van Reekum et al. [63] reported phobic anxiety. The participant's phobias, however, were reported to have pre-dated the TBI. The highest occurrence of specific phobia was reported by Mayou et al. [55] at 7\% in a sample of motor-vehicle accident victims $(n=188)$. It is inferred by descriptives that the majority of the sample evaluated had sustained mild TBIs. None of the phobias were pre-existing and all were related to travel. The notable difference in prevalence in the Mayou et al. study and the two aforementioned studies may be attributable to the samples used; Mayou et al. examined victims of motor-vehicle accidents while the other studies included participants who had incurred injuries from a range of precipitating situations. Perhaps the circumstances under which one's TBI is sustained impact the potential development of a specific phobia. No studies have investigated such a vein, which perhaps may be impeded by low prevalence rates. Considerable diagnostic cross-over may also be a confounding presence, given that avoidance of traumarelated stimuli in PTSD and a specific phobia of driving present significant overlap and a high potential for misdiagnosis.

\section{Social phobia}

Social phobia is characterized by clinically significant anxiety brought on by certain types of social or performance situations and often centres around a fear of negative evaluation from others [23]. Generally surfacing in an individual's mid-teens, social phobia has a lifetime prevalence rate of $12.1 \%$ [19]. The most common fears include public speaking, speaking with strangers and meeting new people. Other anxiety-provoking situations include dating, parties, writing or eating in public and using public restrooms (for reasons of embarrassment) [23].

Social phobia and mild TBI

Despite its status as the most common of all anxiety disorders, social phobia has received very little attention in TBI research. There is currently no literature specifically focused on its study and only peripheral data are available. Using this indirect data, however, a cursory clinical picture of social phobia and mild TBI can be assembled. Those who have sustained a mild TBI often struggle with an adjustment of self-image which is often, at least initially, quite negative [20]. The impact of physical disfigurement or handicap has obvious potential for one to feel uneasy or self-conscious in social situations. It has been noted that those with 
more 'invisible' neuropsychological symptoms such as attention and memory difficulties may be particularly vulnerable to producing anxiety in social situations $[22,70]$. Social gatherings and performance situations rely heavily on such cognitive abilities and can leave one feeling fearful of public acknowledgement of such impairments [20]. Concern regarding a lack of understanding from others and fear of potential embarrassment are also likely sources of anxiety.

No direct prevalence rates in those with TBI presently exist in the literature. Several studies present information regarding pre-morbid psychiatric disorders, yet none provide statistics regarding social phobia. It is unclear if such data are absent due to non-existent prevalence rates of social phobia or lack of specific evaluation. The former is unlikely. Dramatic changes in one's social functioning following a mild TBI have been well documented. Disruptions in social interactions, leisure activities, independence and work status have been noted in those with mild TBI [71]. Research also indicates that those who have sustained a TBI engage in fewer social encounters and have fewer friends than controls, even 2 years post-injury [3]. A sole estimate of the prevalence of acquired social phobia may be inferred based on data collected by van Reekum et al. [63]. Seventeen per cent of their sample were reported to have avoidant personality disorder, a diagnosis reported to have co-morbidity rates with social phobia as high as $89 \%$ [72].

Although the authors also evaluated for Axis I disorders, no diagnoses of social phobia were documented and its absence not addressed. Diagnoses, however, were based on the Diagnostic and Statistical Manual 3rd Edition (DSM-III) [73] which does not allow for comorbid diagnoses of social phobia and avoidant personality disorder. If the presence of social phobia were not assessed, it is possible that it could have been misdiagnosed as avoidant personality disorder. The authors do not provide enough data to confirm or dismiss this speculation. Additional research is needed to clarify this issue in general.

Also of note, poor mild-TBI outcome has been noted in individuals with perfectionistic standards and a tendency for dichotomous thinking, frequent characteristics of those with social phobia [74]. It is Moore et al. impossible to draw any firm conclusions from such indirect data, but such evidence clearly suggests that further investigation is warranted. With such a notable prevalence rate, it would seem logical to assume that social phobia is a diagnosis well represented within mild TBI samples and should be acknowledged. Conversely, if prevalence rates are substantially lower than expected, such data are meaningful and should be further examined. For example, Boker et al. [31] reported that participants with brain injuries localized in the right hemisphere endorse fewer anxiety symptoms than would be expected, often exhibiting indifference and lack of insight. An understanding of how such an injury affects pre-morbid social phobia could shed light on etiology and inform treatment research.

\section{Impacts of anxiety}

\section{Neuropsychological functioning}

Currently the literature offers five studies which examine the impact of anxiety on neuropsychological functioning following mild TBI. The data on this topic have generally been obtained as a less primary directive in a study concerned with other objectives. Nonetheless, the data that do exist offer a peek at what will hopefully, in the near future, elicit more attention and empirical investigation. MacNiven and Finlayson [35], for example, found a significant relationship between scores on the MMPI [38] psychasthenia scale, which measures symptoms similar to those in OCD, and performance on the Category Test [75], a measure of abstract reasoning and concept formation $(n=59)$. The remainder of available data in this vein of research are almost exclusively limited to OCD and PTSD. OCD acquired following a mild TBI has been correlated with deficits in visual-spatial and verbal memory, verbal attention span and fluency and impairment of frontal lobe functioning $[64,66]$. PTSD precipitated by a mild TBI has been correlated with impaired performance on the 30-minute delayed reproduction of the Rey-Osterreith Complex Figure [76], a measure of non-verbal memory, and the Wisconsin Card-Sorting Task [77, $78]$, a measure of flexibility in problem-solving $(n=69)$ [46]. It has been suggested that poor performance on cognitive measures for individuals with mild TBI and PTSD may be misattributed to the mild TBI when the impact of PTSD is the actual source [79]. With anxiety states known to impair attention and memory in the general population [79], it stands to reason that this phenomenon is generalizable to individuals who have sustained a mild TBI. Future research into the neuropsychological functioning of those with mild TBI should incorporate, at the very least, a basic measure of anxiety (as well as depression) into their assessment in order to account for a larger degree of variance and to engender more thoroughly informed conclusions.

\section{Course of recovery}

The presence of psychiatric conditions has been identified as 'a major determinant in outcome after mild TBI' ([16], p. 230). Despite the consistent data that indicate that psychological impairment is detrimental to an individual's course of recovery, few investigators have examined this phenomenon. Those who have do not delineate between anxiety and depression, most 
often investigating general psychiatric morbidity. In general, those with a mild TBI and co-morbid anxiety, as compared to those without, have been reported to be more functionally disabled and to perceive their level of cognitive impairment and injuries to be more severe than objectively evident [68]. In one group of 80 participants with mild TBI, two-thirds of those with a psychiatric diagnosis had prolonged and complicated recoveries, as opposed to the majority of those without psychiatric issues who made much swifter recoveries over the course of 3 months [15]. Fenton et al. [80] found that $39 \%$ of participants with mild TBIs were diagnosed as 'psychiatric cases' at 6 weeks post-injury $(n=45)$. At 6 months, approximately half of these participants endorsed symptoms of PCS and displayed four times the average level of chronic social difficulties than controls. There are also data that indicate that the psychiatric results of a mild TBI stubbornly remain years post-injury, interact with post-concussional symptoms and do not mitigate in the absence of treatment [81]. Merskey [82] found that, 4 years post-injury, close to half of their mild TBI sample who reported psychiatric sequelae showed no improvement in PCS symptoms $(n=27)$.

There is a great need for future studies to focus on the development and validation of interventions to improve prognosis of individuals with mild TBI and difficulties with anxiety. It would be beneficial for future studies to more adequately delineate among the array of psychiatric symptoms and disorders, rather than using overly general categories such as 'psychiatric cases.' Also, although it is sound practice for clinical researchers to use clean samples, this ironically often weeds out those most likely in sustain a mild TBI (those with substance use issues, pre-morbid psychiatric diagnoses, etc.). Generalizability to a more realistically representative group of those with mild TBIs likely suffers as a result. Future treatment-oriented studies may wish to consider this issue, perhaps even comparing the two samples (selective vs. naturalistic) to address whether there is a significant differential impact on data. Perhaps a compromise between empirically sound method and clinically generalizable data can be achieved.

\section{Conclusions}

Overall, the mild TBI research struggles with inconsistencies concerning prevalence rates, the weight and implications of this issue and, in the case of PTSD, even whether certain diagnoses can exist at all. Regardless, it is difficult to ignore the range of studies that document the presence of acquired anxiety disorders, some reported at markedly higher rates than in community samples. This trend seems to vary greatly among spe- cific anxiety disorders, with OCD and PTSD accumulating the bulk of the research in this area. Social phobia, specific phobia and PD with agoraphobia seem to have been overlooked in the body of research on a surprisingly consistent basis.

Gray and McNaughton's [24] model of the neuropsychology of anxiety, as well as the hypotheses and data on the etiology of acquired anxiety disorders specific to mild TBI, provide an important, albeit inconclusive, foundation upon which the findings of a range of research can be conceptualized in a more complete way. There appears to be a relatively distinct split between researchers investigating organic etiology and those investigating functional outcome. It is hoped that future research will ameliorate this situation with perhaps a greater degree of collaboration across disciplines of neuroscience.

Data on the impact of anxiety on neuropsychological symptoms and course of recovery in those with a mild TBI is limited and often overly general, failing to differentiate between psychiatric conditions. Despite such shortcomings, the meager existing data do suggest the presence of a meaningful and detrimental interaction between anxiety and outcome of mild TBI. Such studies establish the importance of further investigation into this vein of research, one which possesses great potential to directly improve the prognosis and treatment of those who have incurred a mild TBI.

\section{Limitations and future directions}

Much of the existing data fall prey to not only traditional methodological issues, but also to pitfalls inherent in the study of mild TBI and its associated constellation of symptoms. The vast majority of studies have no control groups. In such instances, it can be assumed that interviewers are not blind to participants' general circumstances, leaving such studies poorly protected from issues such as experimenter expectancies, especially when diagnostic interviews and subjective clinical judgement are involved. It has been suggested that spinal cord injury patients may be a particularly appropriate control population provided that careful attention is given to weeding those out who may have sustained concomitant head injury [2].

Additionally, significant systematic bias in longitudinal outcome studies has been noted. Corrigan et al. [83] found that a history of alcohol abuse and intoxication at the time of the injury were strongly correlated with loss of follow-up status 1 year post-TBI. Such selective dropout patterns are seldom addressed in the present literature, despite the value and implications of the data. Indeed, if substance abuse is so strongly linked to such a trend, it stands to reason that other disorders may be vulnerable to similar behavior. With high comorbidity with anxiety, studies who have fallen vic- 
tim to substance abuse dropouts are primed to miss a significant number of co-existing anxiety diagnoses. A large-scale study conducted by Brown et al. [84] indicated that those with PD with agoraphobia and PTSD, in particular, demonstrated notably elevated rates of co-morbid substance use disorders $(18 \%$ and $30 \%$, respectively), as compared to community samples [19].

The vast majority of studies do not include data from collateral sources. Research indicates that there is a significant difference in the number of psychiatric symptoms participants report and those reported by collateral sources such as family members [85]. While selfreport of somatic symptoms has been found to be reliably confirmed by other sources, psychiatric symptoms were significantly under-reported by those with TBI, perhaps due to insight and self-awareness difficulties [85]. Such data indicate a potential under-diagnosis of psychiatric disorders in those with mild TBI and highlight the need for inclusion of collateral sources in future research.

As previously mentioned, perhaps an even greater threat to the validity of diagnostic prevalence with mild TBI is the marked overlap between the criteria for anxiety disorders and the symptoms typically experienced during PCS. Symptoms such as impaired concentration and memory, sleep disturbance, anxiety, irritability, fatigue, dizziness, increased sensitivity to noise and a heightened startle reflex are characteristic of both PCS and several anxiety disorders [20]. This extensive overlap has likely led to both the over-diagnosis and the much more likely under-diagnosis of anxiety disorders in those with mild TBI. To complicate the issue, many argue that the PCS itself is not clinically valid [86]. Further research is clearly warranted to tease out these diagnostic confounds by, for example, clarifying more subtle differences in the expression of such symptoms and identifying the potential etiological differential between PCS and anxiety symptoms.

Perhaps the issue which precedes all others is the continued absence of a transdiscipline definition of mild TBI. Indeed, deviations are even present within disciplines, including the studies reviewed in this paper. For example, Mooney and Speed [15] made good use of the criteria outlined by the ACRM to determine inclusion into their mild TBI sample, while Deb et al. [62] solely relied on a GCS of 13-15. Mittenberg et al. [87] considered those who were alert and responsive within 30 minutes of their trauma to be mildly injured, while Koponen et al. [21] required a LOC lasting less than 1 hour. Not only must neuroscience settle on a unified definition of mild TBI, it must be embraced by clinicians and researchers alike if empirical data are to be generalizable and clinically useful.

Arguably, the present literature raises more questions than it answers; much ground has yet to be cov- ered. Areas that warrant a much greater empirical focus are the impacts of anxiety on neuropsychological functioning and outcome, etiology of acquired anxiety (both organic and psychogenic) and data specific to the occurrence of social phobia and co-morbid PD and agoraphobia precipitated by a mild TBI. As previously mentioned, it is recommended that future research focus less of its efforts on diagnostic technicalities, such as those featured in the PTSD controversy, and more on the quantification and expression of specific symptoms and their functional correlates.

\section{Acknowledgements}

Special thanks are extended to the Madonna Rehabilitation Hospital for offering the generous use of its resources and for its continued support of research. Gratitude is also expressed to Heath Raymond for his immeasurable support.

\section{References}

1. Rapoport M, McCauley S, Levin H, Song J, Feinstein A. The role of injury severity in neurobehavioral outcome 3 months after traumatic brain injury. Neuropsychiatry, Neuropsychology, and Behavioral Neurology 2002; 15: 123132.

2. van Reekum R, Cohen T, Wong J. Can traumatic brain injury cause psychiatric disorders? Journal of Clinical Neuroscience 2000; 12: 316-327.

3. Morton MV, Wehman P. Psychosocial and emotional sequelae of individuals with traumatic brain injury: A literature review and recommendations. Brain Injury 1995; 9: 81-92.

4. Ruff RM, Jurica P. In search of a unified definition for mild traumatic brain injury. Brain Injury 1999; 13: 943-952.

5. Centers for Disease Control and Prevention. Heads up: Facts for physicians about mild traumatic brain injury (MTBI); 2004. Available online at: url: http://www.cdc. gov/Migrated_Content/Brochures_and_Catalogs/tbi_ mtbi_facts_for_physicians.pdf

6. Kay T, Harrington DE, Adams R, Mild Traumatic Brain Injury Committee, American Congress of Rehabilitation Medicine, Head Injury Interdisciplinary Special Interest Group. Definition of mild traumatic brain injury. Journal of Head Trauma Rehabilitation 1993; 8: 86-87.

7. Hibbard MR, Uysal S, Kepler K, Bogdany J, Silver J. Axis I psychopathology in individuals with traumatic brain injury. Journal of Head Trauma Rehabilitation 1998; 13: 24-39.

8. Tellier A, Della Malva LC, Winn AC, Grahovac S, Morris $\mathrm{W}$, Brennan-Barnes $\mathrm{M}$. Mild head injury: A misnomer. Brain Injury 1999; 13: 463-475.

9. Gerberding JL, Binder S. Report to Congress on mild traumatic brain injury in the United States: Steps to prevent a serious public health problem. National Center for Injury Prevention and Control. Atlanta: Centers for Disease Control and Prevention; 2003.

10. Bernstein DA. Recovery from mild head injury. Brain Injury 1999; 13: 151-172.

11. Weight DG. Minor head trauma. The Psychiatric Clinics of North America 1998; 3: 609-624. 
12. Rao V, Lyketsos C. Psychiatric aspects of traumatic brain injury. The Psychiatric Clinics of North American 2002; 25: 43-69.

13. McGuire LM, Burright RG, Williams R, Donovick PJ. Prevalence of traumatic brain injury in psychiatric and nonpsychiatric subjects. Brain Injury 1998; 12: 207-214.

14. Burg JS, McGuire LM, Burright RG, Donovick PJ. Prevalence of traumatic brain injury in an inpatient psychiatric population. Journal of Clinical Psychology in Medical Settings 1996; 3: 243-251.

15. Mooney G, Speed J. The association between mild traumatic brain injury and psychiatric conditions. Brain Injury 2001; 15: 865-877.

16. Mooney G, Speed J. Differential diagnosis in mild brain injury: understanding the role of non-organic conditions. Neurorehabilitation 1997; 8: 223-233.

17. Uzzell BP. Mild traumatic brain injury: Much ado about something. In: Varney NR, Roberts RJ, editors. The Evaluation and Treatment of Mild Traumatic Brain Injury. Mahwah, N.J.: Lawrence Erlbaum; 1999, pp. 1-14.

18. Barlow DH (ed.). The experience of anxiety: Shadow of intelligence or specter of death? In: Anxiety and Its Disorders: The Nature and Treatment of Anxiety and Panic. 2nd ed. New York: Guilford Press; 2001, pp. 1-36.

19. Kessler RC, Berglund P, Demler O, Jin R, Walters EE. Lifetime prevalence and age-of-onset distributions of DSMIV disorders in the national comorbidity survey replication. Archives of General Psychiatry 2005; 62: 593-602.

20. Epstein RS, Ursano RJ. Anxiety disorders. In: Silver JM, Yudofsky SC, Hales RE, editors. Neuropsychiatry of Traumatic Brain Injury. Washington, DC: American Psychiatric Press, Inc.; 1994, pp 3-41.

21. Koponen S, Taiminen T, Portin R, Himanen L, Isoniemi H, Heinonen H, Hinkka S, Tenovuo O. Axis I and II psychiatric disorders after traumatic brain injury: a 30-year follow-up study. American Journal of Psychiatry 2002; 159: 1,315-1,321.

22. Hiott DW, Labbate L. Anxiety disorders associated with traumatic brain injuries. Neurorehabilitation 2002;17:345-355.

23. American Psychiatric Association. Diagnostic and Statistical Manual of Mental Disorders. 4th ed., text revision. Washington, D.C.: American Psychiatric Association; 2000.

24. Gray JA, McNaughton N. The neuropsychology of anxiety: Reprise. In: Hope DA, editor. Perspectives on Anxiety, Panic, \& Fear. Lincoln, Neb.: University of Nebraska Press; 1996, pp. 61-134.

25. Drummond LM, Gravestock S. Delayed emergence of obsessive-compulsive neurosis following head injury. British Journal of Psychiatry 1988; 153: 839-842.

26. Williams DJ, Scott JG, Adams RL. Traumatic brain injury. In: Adams RL, Parsons OA, Culbertson JL, Nixon SJ, Russen LA, editors. Neuropsychology for Clinical Practice: Etiology, Assessment, and Treatment of Common Neurological Disorders. Washington, D.C.: American Psychological Association; 1996, pp. 9-64.

27. Cassidy JW. Neuropathology. In: Silver JM, Yudofsky SC, Hales RE, editors. Neuropsychiatry of Traumatic Brain Injury. Washington, D.C.: American Psychiatric Press, Inc.; 1994, pp. 43-79.

28. Jacobson RR. The post-concussional syndrome: physiogenesis, psychogenesis and malingering: an integrative model. Journal of Psychosomatic Research 1995; 39: 675-693.
29. Lezak MD. Relationships between personality disorders, social disturbances, and physical disability following traumatic brain injury. Journal of Head Trauma Rehabilitation 1987; 2: 57-59.

30. Ruff RM, Camenzuli L, Mueller J. Miserable minority: Emotional risk factors that influence the outcome of a mild traumatic brain injury. Brain Injury 1996; 10: 551-565.

31. Boker LL, Butler R, Fleminger S. Are neuropsychiatric symptoms associated with evidence of right brain injury in referrals to a neuropsychiatric brain injury unit? Brain Injury 2001; 15: 65-69.

32. Widiger TA. Mental disorders as discrete clinical conditions: Dimensional versus categorical classification. In: Turner SM, Hersen H, editors. Adult Psychopathology and Diagnosis. 3rd ed. New York: John Wiley \& Sons, Inc.; 1997, pp. 3-23.

33. Schoenhuber R, Gentilini M. Anxiety and depression after mild head injury: a case control study. Journal of Neurology, Neurosurgery, and Psychiatry 1988; 51: 722-724.

34. Speilberger CD, Gorsuch RL, Lushene SH. Manual for the State-Trait Anxiety Inventory. Palo Alto, Calif.: Consulting Psychologist Press; 1970.

35. MacNiven E, Finlayson MA. The interplay between emotional and cognitive recovery after closed head injury. Brain Injury 1993; 7: 241-246.

36. Burke JM, Smith SA, Imhoff CL. The response styles of post-acute traumatic brain-injured patients on the MMPI. Brain Injury 1989; 3: 35-40.

37. Hathaway SR, McKinley JC. The Minnesota Multiphasic Personality Schedule. Minneapolis: University of Minnesota Press; 1943.

38. Graham JR. MMPI-2: Assessing Personality and Psychopathology. 3rd ed. New York: Oxford University Press; 2000.

39. Beck AT. Beck Anxiety Inventory (BAI). San Antonio, Tex.: Psychological Corporation; 1993.

40. Millon T, Millon C, Davis R. Millon Clinical Multiaxial Inventory-III. Minneapolis, Minn.: National Computer Systems; 1994.

41. Stavrakaki C, Vargo B. The relationship of anxiety and depression: a review of the literature. British Journal of Psychiatry 1986; 149: 7-16.

42. Jorge RE, Robinson RG, Starkstein SE, Arndt SV. Depression and anxiety following traumatic brain injury. Journal of Neuropsychiatry 1993; 5: 369-374.

43. Koren D, Arnon I, Klein E. Acute stress response and posttraumatic stress disorder in traffic accident victims: a one-year prospective, follow-up study. American Journal of Psychiatry 1999; 156: 367-373.

44. Bryant RA, Harvey AG. The influence of traumatic brain injury on acute stress disorder and post-traumatic stress disorder following motor vehicle accidents. Brain Injury 1999; 13: 15-22.

45. Feinstein A, Hershkop S, Jardine A, Ouchterloney D. The prevalence and neuropsychiatric correlates of posttraumatic stress symptoms following mild traumatic brain injury. Brain and Cognition 2000; 44: 78-82.

46. Levin HS, Brown SA, Song JX, McCauley SR, Boake C, Contant CR, Goodman H, Kotrla KJ. Depression and posttraumatic stress disorder at three months after mild to moderate traumatic brain injury. Journal of Clinical and Experimental Neuropsychology 2001; 23: 754-769. 
47. Harvey AG, Bryant RA. Predictors of acute stress following mild traumatic brain injury. Brain Injury 1998; 12: 147154.

48. Ohry A, Rattok J, Solomon Z. Post-traumatic stress disorder in brain injury patients. Brain Injury 1996; 10: 687-695.

49. Turnbull SJ, Campbell EA, Swann IJ. Post-traumatic stress disorder symptoms following a head injury: Does amnesia for the event influence the development of symptoms? Brain Injury 2001; 15: 775-785.

50. Warden DL, Labbate LA, Salazar AM, Nelson R, Sheley E, Staudenmeier J, Martin E. Posttraumatic stress disorder in patients with traumatic brain injury and amnesia for the event? Journal of Neuropsychiatry and Clinical Neurosciences 1997; 9: 18-22.

51. American Psychiatric Association. Diagnostic and Statistical Manual of Mental Disorders. 3rd ed., revised. Washington, D.C.: American Psychiatric Association; 1987.

52. Bryant RA, Marosszeky JE, Crooks J, Baguley I, Gurka J. Coping style and post-traumatic stress disorder following severe traumatic brain injury. Brain Injury 1999; 14: 175-180.

53. Blanchard EB, Hickling EJ, Taylor AE, Loos WR. Psychiatric morbidity associated with motor vehicle accidents. Journal of Nervous and Mental Disease 1995; 8: 495-504.

54. Sbordone RL, Liter JC. Mild traumatic brain injury does not produce post-traumatic stress disorder. Brain Injury 1995; 9: 405-412.

55. Mayou R, Bryant B, Duthie R. Psychiatric consequences of road traffic accidents. British Medical Journal 1993; 307: 647-651.

56. McMillan TC. Errors in diagnosing post-traumatic stress disorder after traumatic brain injury. Brain Injury 2001; 15: 39-46.

57. Ruff RM. Discipline-specific approach versus individual care. In: Varney NR, Roberts RJ, editors. The Evaluation and Treatment of Mild Traumatic Brain Injury. Mahwah, N.J.: Lawrence Erlbaum; 1999, pp 99-114.

58. Adler A. Neuropsychiatric complications in victims of Boston's Coconut Grove disaster. Journal of the American Medical Association 1943; 123: 1,098-1,101.

59. Bryant RA. Posttraumatic stress disorder, flashbacks, and pseudomemories in closed head injury. Journal of Traumatic Stress 1996; 9: 621-629.

60. King NS. Post-traumatic stress disorder and head injury as a dual diagnosis: 'Islands' of memory as a mechanism. Journal of Neurology, Neurosurgery, and Psychiatry 1997; 62: 82-84.

61. Gronwall D, Wrightson P. Duration of post-traumatic amnesia after mild head injury. Journal of Clinical Neuropsychology 1980; 2: 51-60.

62. Deb S, Lyons I, Koutzoukis C, Ali I, McCarthy G. Rate of psychiatric illness 1 year after traumatic brain injury. American Journal of Psychiatry 1999; 44: 374-378.

63. van Reekum R, Bolago I, Finlayson S, Garner S, Links PS. Psychiatric disorders after traumatic brain injury. Brain Injury 1996; 10: 319-327.

64. Kant R, Smith-Seemiller L, Duffy JD. Obsessive-compulsive disorder after closed head injury: review of literature and report of four cases. Brain Injury 1996; 10: 55-63.

65. McKeon J, McGuffin P, Robinson P. Obsessive-compulsive neurosis following head injury: a case report of four cases. British Journal of Psychiatry 1984; 144: 190-192.
66. Berthier ML, Kulisevsky J, Gironell A, Heras JA. Obsessive-compulsive disorder associated with brain lesions: clinical phenomenology, cognitive function, and anatomic correlates. Neurology 1996; 47: 353-360.

67. Scheutzow MH, Wiercisiewski DR. Panic disorder in a patient with traumatic brain injury: a case report and discussion. Brain Injury 1999; 13: 705-714.

68. Fann JR, Katon WJ, Uomoto JM, Esselman PC. Psychiatric disorders and functional disability in outpatients with traumatic brain injuries. American Journal of Psychiatry 1995; 152: 1,493-1,499.

69. Kessler RC, McGonagle KA, Zhao S, Nelson C, Hughes M, Eshleman S, Wittchen H, Kendler K. Lifetime and 12-month prevalence of DSM-III-R psychiatric disorders in the United States: results from the National Comorbidity Survey. Archives of General Psychiatry 1994; 51: 8-19.

70. Wright JC, Telford R. Psychological problems following minor head injury: a prospective study. British Journal of Clinical Psychology 1996; 35: 399-412.

71. Antonak RF, Livneb H, Antonak C. A review of research on psychosocial adjustment to impairment in persons with traumatic brain injury. Journal of Head Trauma Rehabilitation 1993; 8: 87-100.

72. Brown EJ, Heimberg RG, Juster HR. Social phobia subtype and avoidant personality disorder: effect on severity of social phobia, impairment, and outcome of cognitive behavioral treatment. Behavior Therapy 1995; 26: 467-486.

73. American Psychiatric Association. Diagnostic and Statistical Manual of Mental Disorders. 3rd ed. Washington, D.C.: American Psychiatric Association; 1980.

74. Turk CL, Heimberg RG, Hope DA. Social anxiety disorder. In: Barlow DH, editor. Clinical Handbook of Psychological Disorders. 3rd ed. New York: Guilford Press; 2001, pp 114-153.

75. Reitan RM, Wolfson D. The Halstead-Reitan Neuropsychological Test Battery: Theory and Clinical Interpretation. Tucson: Neuropsychology Press; 1986.

76. Rey A. L'examen psychologique dans les cas d'encéphalopathie truamatique. Archives de Psychologie 1941; 28: 286340 .

77. Grant DA, Berg EA. A behavioral analysis of degree of impairment and ease of shifting to new responses in a Weigl-type card sorting problem. Journal of Experimental Psychology 1948; 39: 404-411.

78. Heaton RK, Chelune GJ, Talley JL, Kay GG, Curtis G. Wisconsin Card Sorting Test (WCST) Manual Revised and Expanded. Odessa, Fla.: Psychological Assessment Resources; 1993.

79. Hovland D, Raskin SA. Anxiety and posttraumatic stress. In: Raskin SA, Mateer CA, editors. Neuropsychological Management of Mild Traumatic Brain Injury. New York: Oxford University Press; 2000, pp 171-183.

80. Fenton G, McClelland R, Montgomery A, MacFlynn G, Rutherford $W$. The postconcussional syndrome: social antecedents and psychological sequelae. British Journal of Psychiatry 1993; 162: 493-497.

81. Bornstein RA, Miller HB, Van Schoor JT. Neuropsychological deficits and emotional disturbance in head-injured patients. Journal of Neurosurgery 1989; 70: 509-513.

82. Mersky H, Woodforde JM. Psychiatric sequelae of mild head injury. Brain 1972; 95: 321-328. 
83. Corrigan JD, Bogner JA, Mysiw J, Clinchot D, Fugate L. Systematic bias in outcome studies of persons with traumatic brain injury. Archives of Physical and Medical Rehabilitation 1997; 78: 132-137.

84. Brown TA, Campbell LA, Lehman CL, Grisham JR, Mancill RB. Current and lifetime comorbidity of the DSM-IV anxiety and mood disorders in a large clinical sample. Journal of Abnormal Psychology 2001; 110: 585-599.

85. Sbordone RJ, Seyranian GD, Ruff RM. Are the subjective complaints of traumatically brain injured patients reliable? Brain Injury 1998; 12: 505-515.

86. Malec JF. Mild traumatic brain injury: Scope of the problem. In: Varney NR, Roberts RJ, editors. The Evaluation and Treatment of Mild Traumatic Brain Injury. Mahwah, N.J.: Lawrence Erlbaum Associates; 1999, pp 15-37.

87. Mittenberg W, DiGiulio DV, Perrin S, et al. Symptoms following mild head injury: expectation as etiology. Journal of Neurology, Neurosurgery, and Psychiatry 1992; 55: 200-204. 